\title{
ASYMPTOTICS FOR THE MINIMUM RIESZ ENERGY AND BEST-PACKING ON SETS OF FINITE PACKING PREMEASURE
}

\author{
SERGIY Borodachov
}

\begin{abstract}
We show that for every compact set $A \subset \mathbb{R}^{m}$ of finite $\alpha$-dimensional packing premeasure $0<\alpha \leq m$, the lower limit of the normalized discrete minimum Riesz $s$-energy $(s>\alpha)$ coincides with the outer measure of $A$ constructed from this limit by method I. The asymptotic behavior of the discrete minimum energy on compact subsets of a self-similar set $K$ satisfying the open set condition is also studied for $s$ greater than the Hausdorff dimension of $K$. In addition, similar problems are studied for the best-packing radius.
\end{abstract}

2010 Mathematics Subject Classification: Primary: 28A70, 28A80; Secondary: 31C99, 74G65.

Key words: minimum Riesz energy, best-packing, $\epsilon$-complexity, packing measure and premeasure, self-similar set, method I.

\section{Introduction}

It is known that the $\alpha$-dimensional packing measure of a compact set $A$ in $\mathbb{R}^{m}$ and its $\alpha$-dimensional packing premeasure will coincide if the packing premeasure of $A$ is finite (see [14]). In order to construct packing measure from packing premeasure a procedure known as method I is used. In this paper we study a similar question for the discrete minimum energy limits, namely, under what conditions the lower limit of the normalized discrete minimum Riesz energy (the quantity $\underline{g}_{s, \alpha}(A)$ defined in (3) below) will coincide with the outer measure of $A$ constructed using method I from the set function $\underline{g}_{s, \alpha}$.

Another result obtained in this paper is related to the results by Kolmogorov and Tikhomirov [27] and by Hardin and Saff [22], [23]. The asymptotic behavior as $\epsilon$ tends to 0 of the $\epsilon$-complexity of a Jordan measurable compact set $A$ in $\mathbb{R}^{d}$ was found in [27, Theorem IX]. The asymptotic behavior as $N$ grows of the minimum Riesz $s$-energy of $N$-points restricted to a compact set $A \subset \mathbb{R}^{d}$ of positive Lebesgue measure $(s>d)$ is also known (see $\left[\mathbf{2 3}\right.$, Theorem 2.2]). A compact set in $\mathbb{R}^{d}$ can be 
placed inside a cube, and the cube in turn, can be considered as a selfsimilar set. We obtain analogs of these results when the compact set $A$ is contained in a given self-similar set in $\mathbb{R}^{d}$ that satisfies the open set condition. Since the upper and the lower limits of the normalized Riesz energy and best-packing may differ on some self-similar sets, we consider the upper and the lower limit separately. Our result may be applied to estimating the Hausdorff probability measure of a compact subset $A$ of a self-similar set $K$. To do this one could minimize on $A$ and on $K$ the Riesz energy of $N$-point configurations for some "large" value of $N$ and the value of $s$ greater than the Hausdorff dimension of $K$ and then find the ratio of the two energies. We remark that methods of computing the Hausdorff and packing measure of a whole self-similar set, which use other ideas were considered in $[\mathbf{3 3}]$.

The paper is structured as follows. In Section 2 we introduce the general notation and definitions. In Section 3 we review known results for the minimum energy and best-packing problem on rectifiable sets. In Section 4 we recall the definitions related to self-similar sets and formulate the asymptotic results for the minimum energy and the best-packing radius on compact subsets of self-similar sets. In Section 5 we formulate the result concerning the outer measures constructed from the limits of the normalized values of the minimum energy and best-packing. In Section 6 we obtain a regularity result, which is used in Sections 7 and 8 to prove the main results of this paper.

\section{Setting of the problems, notation and definitions}

Let $m \in \mathbb{N}$ and $s>0$. For an arbitrary collection of points $\omega_{N}:=$ $\left\{x_{1}, \ldots, x_{N}\right\} \subset \mathbb{R}^{m}, N \in \mathbb{N}, N \geq 2$, its Riesz $s$-energy is defined to be

$$
E_{s}\left(\omega_{N}\right):=\sum_{1 \leq i \neq j \leq N} \frac{1}{\left|x_{i}-x_{j}\right|^{s}}
$$

where $|\cdot|$ stands for the Euclidian distance in $\mathbb{R}^{m}$. The discrete minimum Riesz $N$-point s-energy of a given set $A$ in $\mathbb{R}^{m}$ is defined as

$$
\mathcal{E}_{s}(A, N):=\inf _{\substack{\omega_{N} \subset A \\ \# \omega_{N}=N}} E_{s}\left(\omega_{N}\right),
$$

where $\# X$ denotes the cardinality of a set $X$. The case of $m=3, s=1$, and $A$ being a sphere in $\mathbb{R}^{3}$ corresponds to the classical Thomson's problem of finding the ground state configurations of $N$ classical electrons on the sphere. For the exact results on Thomson's problem and further reviews see $[\mathbf{4 3}],[\mathbf{3}],[\mathbf{2 8}],[\mathbf{9}]$, and references therein. 
In known cases when $s$ is greater than the Hausdorff dimension of $A$, interactions between points located close to each other on $A$ determine the main term of the energy sum as $N \rightarrow \infty$. This case is referred to as the case of short range interactions on $A$.

The limiting case of the minimum $s$-energy problem as $s \rightarrow \infty$, is the best-packing problem, which we will state next. For a configuration $\omega_{N}=\left\{x_{1}, \ldots, x_{N}\right\} \subset \mathbb{R}^{m}$, denote

$$
\delta\left(\omega_{N}\right):=\min _{1 \leq i \neq j \leq N}\left|x_{i}-x_{j}\right|
$$

The best-packing $N$ point distance on the set $A$ is defined as

$$
\delta_{N}(A):=\sup _{\substack{\omega_{N} \subset A \\ \# \omega_{N}=N}} \delta\left(\omega_{N}\right) .
$$

A configuration $\bar{\omega}_{N} \subset A$ attaining the supremum on the right-hand side of (2) is called a best-packing configuration. Without loss of generality, we can consider only compact sets $A$ in both problems, since for an unbounded set, the minimal energy is zero and the best-packing distance equals infinity. Moreover, for any set and its closure, the minimal energies have the same value and the best-packing distances coincide as well. When $A$ is compact, there always exists an optimal $N$-point configuration in (1) and (2) and the infimum (supremum) can be replaced by the minimum (maximum). Formulated for the sphere in $\mathbb{R}^{3}$ the problem of finding quantity (2) is known as the Tammes problem. For notable results and reviews on this problem see [13], $[\mathbf{3 7}],[\mathbf{1 0}],[\mathbf{4}],[\mathbf{2 1}]$, and $[\mathbf{1 5}]$. It is not difficult to see that for every $N$ fixed,

$$
\lim _{s \rightarrow \infty} \mathcal{E}_{s}(A, N)^{1 / s}=\frac{1}{\delta_{N}(A)} .
$$

The best-packing problem is dual to the $\epsilon$-complexity problem, which was first considered in [26] and [27]. For every $\epsilon>0$, the $\epsilon$-complexity $C_{\epsilon}(A)$ of a compact set $A \subset \mathbb{R}^{m}$ is defined as

$$
C_{\epsilon}(A)=\max \{\# X: X \subset A, \quad \text { and } \quad \forall x \neq y \in X,|x-y| \geq \epsilon\}
$$

The quantity $\log _{2} C_{\epsilon}(A)$ is known as $\epsilon$-capacity of the set $A$ and finds its applications in the information theory. The problem about $\epsilon$-complexity also has applications in the theory of dynamical systems (see for example [1] and [2]). 
Let $0<\alpha \leq m$ and $s>\alpha$. Denote

$$
\underline{g}_{s, \alpha}(A):=\liminf _{N \rightarrow \infty} \frac{\mathcal{E}_{s}(A, N)}{N^{1+s / \alpha}}, \quad \bar{g}_{s, \alpha}(A):=\limsup _{N \rightarrow \infty} \frac{\mathcal{E}_{s}(A, N)}{N^{1+s / \alpha}},
$$

and

$$
g_{s, \alpha}(A):=\lim _{N \rightarrow \infty} \frac{\mathcal{E}_{s}(A, N)}{N^{1+s / \alpha}},
$$

provided that the limit in (4) exists. For every $\alpha \in(0, m]$, let

$$
\begin{aligned}
& \underline{g}_{\infty, \alpha}(A):=\liminf _{N \rightarrow \infty} \delta_{N}(A) \cdot N^{1 / \alpha}=\liminf _{\epsilon \rightarrow 0} \epsilon \cdot C_{\epsilon}(A)^{1 / \alpha}, \\
& \bar{g}_{\infty, \alpha}(A):=\limsup _{N \rightarrow \infty} \delta_{N}(A) \cdot N^{1 / \alpha}=\limsup _{\epsilon \rightarrow 0} \epsilon \cdot C_{\epsilon}(A)^{1 / \alpha} .
\end{aligned}
$$

Denote also

$$
g_{\infty, \alpha}(A):=\lim _{N \rightarrow \infty} \delta_{N}(A) \cdot N^{1 / \alpha}=\lim _{\epsilon \rightarrow 0} \epsilon \cdot C_{\epsilon}(A)^{1 / \alpha},
$$

provided that these limits exist. We remark that

$$
\text { (6) } \lim _{s \rightarrow \infty}\left(\underline{g}_{s, \alpha}(A)\right)^{1 / s}=\frac{1}{\bar{g}_{\infty, \alpha}(A)} \quad \text { and } \quad \lim _{s \rightarrow \infty}\left(\bar{g}_{s, \alpha}(A)\right)^{1 / s}=\frac{1}{\underline{g}_{\infty, \alpha}(A)} \text {. }
$$

The proof of this fact can be found, for example, in [7]. We say that a sequence of configurations $\omega_{N}=\left\{x_{1, N}, \ldots, x_{N, N}\right\}, N \in \mathbb{N}, N \geq 2$, on $A$ is asymptotically distributed according to a given Borel probability measure $\mu$ supported on $A$, if for every Borel subset $B$ of $A$ whose boundary relative to $A$ has $\mu$-measure zero, there holds

$$
\lim _{N \rightarrow \infty} \frac{\#\left(\omega_{N} \cap B\right)}{N}=\mu(B) .
$$

In this case we will write

$$
\nu\left(\omega_{N}\right) \stackrel{*}{\rightarrow} \mu, \quad N \rightarrow \infty .
$$

A sequence $\left\{\omega_{N}\right\}_{N \in \mathbb{N}}$ of $N$-point configurations on $A$ is called asymptotically s-energy minimizing, if

$$
\lim _{N \rightarrow \infty} \frac{E_{s}\left(\omega_{N}\right)}{\mathcal{E}_{s}(A, N)}=1,
$$

and it is called asymptotically best-packing if

$$
\lim _{N \rightarrow \infty} \frac{\delta\left(\omega_{N}\right)}{\delta_{N}(A)}=1 .
$$


Let $\mathcal{H}_{\alpha}, 0<\alpha \leq m$, be the $\alpha$-dimensional Hausdorff measure in $\mathbb{R}^{m}$ and let $\operatorname{dim} A$ be the Hausdorff dimension of the set $A$. In what follows, when $\alpha=d \in \mathbb{N}$, the measure $\mathcal{H}_{d}$ will be normalized so that the isometric copy of the cube $[0,1]^{d}$ embedded in $\mathbb{R}^{m}$ has measure 1 . Given a compact set $A \subset \mathbb{R}^{m}$ with $0<\mathcal{H}_{\lambda}(A)<\infty, 0<\lambda \leq m$, let $h_{\lambda}=h_{\lambda, A}$ be the probability measure supported on $A$ such that for every $\mathcal{H}_{\lambda}$-measurable subset $B \subset A$,

$$
h_{\lambda, A}(B):=\frac{\mathcal{H}_{\lambda}(B)}{\mathcal{H}_{\lambda}(A)} .
$$

A set $A \subset \mathbb{R}^{m}$ is called $d$-rectifiable, $d \in \mathbb{N}, d \leq m$, if it is an image of a bounded set from $\mathbb{R}^{d}$ with respect to some Lipschitz mapping $\varphi: \mathbb{R}^{d} \rightarrow$ $\mathbb{R}^{m}$.

\section{Known asymptotic results for rectifiable sets}

For a closed $d$-rectifiable set $A \subset \mathbb{R}^{m}$ with $\mathcal{H}_{d}(A)>0$, the minimal discrete $s$-energy has the following asymptotic behavior (cf. $[\mathbf{2 2}]$ or $[\mathbf{2 3}]$ and $[\mathbf{5}])$ :

$$
g_{s, d}(A)=\lim _{N \rightarrow \infty} \frac{\mathcal{E}_{s}(A, N)}{N^{1+s / d}}=\frac{C_{s, d}}{\mathcal{H}_{d}(A)^{s / d}}, \quad s>d .
$$

For the best-packing distance and the $\epsilon$-complexity of $A$, we also have

$$
g_{\infty, d}(A)=C_{\infty, d} \mathcal{H}_{d}(A)^{1 / d},
$$

see [27] for the case $d=m$ and $A$ being Jordan measurable and [7] for the case $d \leq m$ and $A$ being $d$-rectifiable. Here $C_{s, d}$ and $C_{\infty, d}$ are positive constants independent of $A$. The sequences of optimal $N$-point configurations for both problems are asymptotically distributed according to the measure $h_{\lambda, A}$. When the compact set $A$ is a countable union of $d$-rectifiable sets, these relations remain true if $\mathcal{H}_{d}(A)$ equals the $d$-dimensional Minkowski content of $A$ (cf. [7]).

We remark that $C_{s, 1}=2 \zeta(s), s>1$, where $\zeta(s)=\sum_{k=1}^{\infty} k^{-s}$ is the classical Riemann zeta-function [31], and $C_{\infty, 1}=1, C_{\infty, 2}=(2 / \sqrt{3})^{1 / 2}[\mathbf{1 3}]$, and $C_{\infty, 3}=\sqrt[6]{2}[\mathbf{2 1}]$. The value of $C_{s, d}$ in all other cases is still unknown (see [8] for estimates for these constants). Asymptotic results for the quantization problem on rectifiable sets were obtained in [36] (see also references therein).

We also remark that in the case of long range interactions, i.e. when $0<s<\operatorname{dim} A$, we always have $\mathcal{E}_{s}(A, N) \asymp N^{2}, N \rightarrow \infty$ (see e.g. [30]). 


\section{Minimal Riesz energy on compact subsets of a self-similar set}

According to relations (7) and (8), restrictions of the set functions $g_{s, d}(\cdot)^{-d / s}$ and $g_{\infty, d}(\cdot)^{d}$ to the class of $d$-rectifiable sets in $\mathbb{R}^{m}$ coincide with constant multiples of the Hausdorff measure. In this section we show that the restrictions of the set functions $g_{s, d}(\cdot)^{-d / s}$ and $g_{\infty, d}(\cdot)^{d}$ to the family of compact subsets of a given self-similar set of Hausdorff dimension $\alpha$ satisfying the open set condition coincide with constant multiples of $\mathcal{H}_{\alpha}$.

Basic definitions and properties. Recall that a mapping $S: \mathbb{R}^{m} \rightarrow$ $\mathbb{R}^{m}$ is called a contracting similitude if there is a constant $\sigma \in(0,1)$ such that for every $x, y \in \mathbb{R}^{m}$,

$$
|S(x)-S(y)|=\sigma|x-y| .
$$

Given a finite system of contracting similitudes $S_{1}, \ldots, S_{p}: \mathbb{R}^{m} \rightarrow \mathbb{R}^{m}$, there exists a unique non-empty compact set $K \subset \mathbb{R}^{m}$ such that

$$
K=\bigcup_{i=1}^{p} S_{i}(K)
$$

see [25]. If with $\lambda=\operatorname{dim} K$ we have $\mathcal{H}_{\lambda}\left(S_{i}(K) \cap S_{j}(K)\right)=0$ for every $i \neq j$, the compact set $K$ is called self-similar (see e.g. [32, p. 67]).

Following [34] we say that a collection of contracting similitudes $S_{1}, \ldots, S_{p}$ satisfies the open set condition (OSC) if there is a non-empty open set $\mathcal{O} \subset \mathbb{R}^{m}$ such that

$$
\bigcup_{i=1}^{p} S_{i}(\mathcal{O}) \subset \mathcal{O}, \quad \text { and } \quad S_{i}(\mathcal{O}) \cap S_{j}(\mathcal{O})=\emptyset, \quad i \neq j .
$$

Self-similar sets, for which (10) holds, have the following properties $[\mathbf{2 5}]$ (this result is also cited in [11] and [32]).

Theorem 1. If a collection of $p$ contracting similitudes with contraction coefficients $\sigma_{1}, \ldots, \sigma_{p}$ satisfies the OSC, then the corresponding compact invariant set $K$ is self-similar, and the Hausdorff dimension of $K$ equals the unique number $\lambda$ such that

$$
\sum_{i=1}^{p} \sigma_{i}^{\lambda}=1
$$

Moreover, we have $0<\mathcal{H}_{\lambda}(K)<\infty$ and there are positive and finite numbers $a$ and $b$ such that

$$
a r^{\lambda} \leq \mathcal{H}_{\lambda}(K \cap B(x, r)) \leq b r^{\lambda}, \quad \text { for every } \quad x \in K, \quad \text { and } \quad 0<r \leq 1 .
$$


It was also proved by Schief [38] that if a system of contracting similitudes $S_{1}, \ldots, S_{p}$ generating a self-similar set $K$, and a positive number $\lambda$ are such that $(11)$ holds and $\mathcal{H}_{\lambda}(K)>0$, then the system $S_{1}, \ldots, S_{p}$ satisfies the OSC.

In what follows, $\lambda$ will denote the Hausdorff dimension of the selfsimilar set $K$.

Known asymptotic results for self-similar sets. The asymptotic behavior of best-packing distance and $\epsilon$-complexity on a self-similar set $K$ satisfying the OSC was studied in the paper [29]. This paper in particular showed that if the contraction coefficients $\sigma_{1}, \ldots, \sigma_{p}$ of the similitudes are such that the additive group generated by $\ln \sigma_{1}, \ldots, \ln \sigma_{p}$ is dense in $\mathbb{R}$, then for $\lambda=\operatorname{dim} K$, we have $0<\underline{g}_{\infty, \lambda}(K)=\bar{g}_{\infty, \lambda}(K)<$ $\infty$.

Paper [29] also proves that any sequence $\left\{\bar{\omega}_{\epsilon}\right\}, \epsilon>0$, of $\epsilon$-complexity configurations on a self-similar set $K$ satisfying the OSC will have limiting measure $h_{\lambda, K}$ as $\epsilon \rightarrow 0$. This does not always hold for sequences of best-packing configurations on $K$ whose cardinality tends to infinity. To construct a counterexample one can take $K$ to be the classical Cantor subset of $[0,1]$ and $\mathcal{N}=\left\{2^{m}+2^{m-1}: m \in \mathbb{N}\right\}$. There exists a sequence $\left\{\omega_{N}\right\}_{N \in \mathcal{N}}$, of best-packing $N$-point configurations on $K$, which has $2^{m}$ points on $K \cap\left[0, \frac{1}{3}\right]$ and $2^{m-1}$ points on $K \cap\left[\frac{2}{3}, 1\right]$ for every $m \in \mathbb{N}$.

Limit (4) does not always exist on self-similar sets. It was shown in [7], that if the contraction coefficients of the similitudes $S_{1}, \ldots, S_{p}$ are the same and the images $S_{1}(K), \ldots, S_{p}(K)$ are pairwise disjoint, then for every $s$ sufficiently large,

$$
0<\underline{g}_{s, \lambda}(K)<\bar{g}_{s, \lambda}(K)<\infty .
$$

Under the assumption that the images $S_{1}(K), \ldots, S_{p}(K)$ are pairwise disjoint, a recent result in $[\mathbf{1 6}]$ finds the cases when the equality $\underline{g}_{s, \lambda}(K)=$ $\bar{g}_{s, \lambda}(K)$ holds and the limiting measure for the optimal configurations is $h_{\lambda, K}$. We remark that the asymptotic quantization problem for selfsimilar probabilities was studied in [18] (see also references therein).

Our result stated below, describes the limits of the normalized minimal energy on compact subsets of $K$.

Theorem 2. Let $K$ be a self-similar set generated by a system of contracting similitudes satisfying the $O S C$ and $s>\lambda=\operatorname{dim} K$. Consider a non-empty compact subset $A$ of $K$. There holds

$$
\liminf _{N \rightarrow \infty} \frac{\mathcal{E}_{s}(A, N)}{N^{1+s / \lambda}}=\frac{\underline{g}_{s, \lambda}(K)}{h_{\lambda, K}(A)^{s / \lambda}}
$$


and

$$
\limsup _{N \rightarrow \infty} \frac{\mathcal{E}_{s}(A, N)}{N^{1+s / \lambda}}=\frac{\bar{g}_{s, \lambda}(K)}{h_{\lambda, K}(A)^{s / \lambda}} .
$$

Assume that $\mathcal{H}_{\lambda}(A)>0$. If $\underline{g}_{s, \lambda}(K)=\bar{g}_{s, \lambda}(K)$, then for any asymptotically s-energy minimizing sequence $\left\{\bar{\omega}_{N}\right\}_{N=2}^{\infty}$ of $N$-point configurations on $A$, we have

$$
\nu\left(\bar{\omega}_{N}\right) \stackrel{*}{\rightarrow} h_{\lambda, A}, \quad N \rightarrow \infty .
$$

In the case $\underline{g}_{s, \lambda}(K)<\bar{g}_{s, \lambda}(K)$ the following holds. If $\mathcal{N} \subset \mathbb{N}$ is an infinite sequence and $\left\{\bar{\omega}_{N}\right\}_{N \in \mathcal{N}}, \# \bar{\omega}_{N}=N, N \in \mathcal{N}$, is a sequence of point configurations on $A$ such that

$$
\lim _{\mathcal{N} \ni N \rightarrow \infty} \frac{E_{s}\left(\bar{\omega}_{N}\right)}{N^{1+s / \lambda}}=\underline{g}_{s, \lambda}(A)
$$

then we have

$$
\nu\left(\bar{\omega}_{N}\right) \stackrel{*}{\rightarrow} h_{\lambda, A}, \quad \mathcal{N} \ni N \rightarrow \infty .
$$

If $\mathcal{H}_{\lambda}(A)=0$, the quantity $h_{\lambda, K}(A)^{-s / \lambda}$ is understood to be $\infty$.

Remark 1. Relations (14) and (15) imply that the ratio $\frac{\bar{g}_{s, \lambda}(A)}{\underline{g}_{s, \lambda}(A)}, s>\lambda$, $\mathcal{H}_{\lambda}(A)>0$, does not depend on the compact set $A$. In particular, if the limit

$$
g_{s, \lambda}(K)=\lim _{N \rightarrow \infty} \frac{\mathcal{E}_{s}(K, N)}{N^{1+s / \lambda}}
$$

exists for a self-similar set $K$ with $\operatorname{dim} K=\lambda$ satisfying the OSC $\left(g_{s, \lambda}(K)\right.$ will then be finite and positive), then the limit $g_{s, \lambda}(A)$ will exist as a finite and positive number for any compact subset $A \subset K$ with $\mathcal{H}_{\lambda}(A)>0$. If the limit $g_{s, \lambda}(K)$ does not exist, then the limit $g_{s, \lambda}(A)$ does not exist for any compact subset $A \subset K$ with $\mathcal{H}_{\lambda}(A)>0$.

Remark 2. If $K$ is a cube in $\mathbb{R}^{d}$ containing the compact set $A$ of positive $d$-dimensional Lebesgue measure, Theorem 2 gives a different method (than the one used in $[\mathbf{2 3}]$ ) to derive (7) for $A$ provided that (7) is proved for $K$.

As we mentioned above, in the paper [29] the limiting distribution of $\epsilon$-complexity configurations is obtained on the whole self-similar set $K$. The statement below gives the asymptotic behavior of best-packing configurations on every compact subset of $K$ of positive $\mathcal{H}_{\lambda}$-measure. 
Corollary 1. Let $K$ be a self-similar set generated by a system of contracting similitudes satisfying the $O S C$ and $\lambda=\operatorname{dim} K$. Consider a non-empty compact subset $A \subset K$. There holds

$$
\underline{g}_{\infty, \lambda}(A)=h_{\lambda, K}(A)^{1 / \lambda} \underline{g}_{\infty, \lambda}(K) \text { and } \bar{g}_{\infty, \lambda}(A)=h_{\lambda, K}(A)^{1 / \lambda} \bar{g}_{\infty, \lambda}(K) .
$$

Assume that $\mathcal{H}_{\lambda}(A)>0$. If $\underline{g}_{\infty, \lambda}(K)=\bar{g}_{\infty, \lambda}(K)$, then for any asymptotically best-packing sequence $\left\{\widetilde{\omega}_{N}\right\}_{N=2}^{\infty}$ of $N$-point configurations on $A$, we have

$$
\nu\left(\widetilde{\omega}_{N}\right) \stackrel{*}{\rightarrow} h_{\lambda, A}, \quad N \rightarrow \infty .
$$

In the case $\underline{g}_{\infty, \lambda}(K)<\bar{g}_{\infty, \lambda}(K)$ the following holds. If $\mathcal{N} \subset \mathbb{N}$ is an infinite sequence and $\left\{\widetilde{\omega}_{N}\right\}_{N \in \mathcal{N}}, \# \widetilde{\omega}_{N}=N, N \in \mathcal{N}$, is a sequence of point configurations on $A$ such that

$$
\lim _{\mathcal{N} \ni N \rightarrow \infty} \delta\left(\widetilde{\omega}_{N}\right) \cdot N^{1 / \lambda}=\bar{g}_{\infty, \lambda}(A),
$$

then we have

$$
\nu\left(\widetilde{\omega}_{N}\right) \stackrel{*}{\rightarrow} h_{\lambda, A}, \quad \mathcal{N} \ni N \rightarrow \infty
$$

\section{The minimum Riesz energy on sets of finite packing premeasure}

This section studies the outer measures constructed using method I from the lower limit of normalized minimal Riesz energy or the upper limit of normalized $\epsilon$-complexity (best-packing distance). Throughout the rest of the paper we will agree that $\underline{g}_{s, \alpha}(\emptyset)^{-\alpha / s}=\bar{g}_{\infty, \alpha}(\emptyset)^{\alpha}=0$.

Basic definitions. Set functions $\underline{g}_{s, \alpha}(A)^{-\alpha / s}, s>\alpha$, and $\bar{g}_{\infty, \alpha}(A)^{\alpha}$ are subadditive (see [23, Lemma 3.2] and [6]), but not countably subadditive since each of these quantities has the same value on a set and its closure and both quantities equal zero on singletons. One can still obtain a countably sub-additive set function from $\underline{g}_{s, \alpha}(A)^{-\alpha / s}$ and $\bar{g}_{\infty, \alpha}(A)^{\alpha}$ by using method I: denote

$$
\nu_{s, \alpha}(A):=\inf \left\{\sum_{i} \underline{g}_{s, \alpha}\left(A_{i}\right)^{-\alpha / s}: A \subset \bigcup_{i} A_{i}\right\}, \quad s>\alpha,
$$

and

$$
\nu_{\infty, \alpha}(A):=\inf \left\{\sum_{i} \bar{g}_{\infty, \alpha}\left(A_{i}\right)^{\alpha}: A \subset \bigcup_{i} A_{i}\right\} .
$$

Recall that a non-negative set function $\nu$ defined on all subsets of $\mathbb{R}^{m}$ is called an outer measure (see e.g. [32]) if 
(1) $\nu(\emptyset)=0$.

(2) For every sets $A, B \subset \mathbb{R}^{m}$ such that $A \subset B$ we have $\nu(A) \leq \nu(B)$.

(3) For any set $A$ and at most a countable collection of sets $\left\{A_{i}\right\}$ in $\mathbb{R}^{m}$ such that $A \subset \bigcup_{i} A_{i}$ we have $\nu(A) \leq \sum_{i} \nu\left(A_{i}\right)$.

Hence, the set function $\nu_{s, \alpha}(A), s \in(\alpha, \infty]$ will be an outer measure. The $\sigma$-algebra of measurable sets with respect to $\nu_{s, \alpha}$, is non-trivial since it contains all sets for which $\nu_{s, \alpha}(A)=0$ and complements of such sets in $\mathbb{R}^{m}$. We do not know if the $\sigma$-algebra of measurable sets in the case $s<\infty$ contains other sets. We remark that this question in the case $s=\infty$ was studied by O. Zindulka.

The idea of defining the dimension of a set using covering by sets of vanishing diameter was first introduced in [35]. The notion of the packing measure recalled below, and the notion of the packing dimension were first studied in the papers $[\mathbf{3 9}]-[\mathbf{4 2}]$. For every set $A \subset \mathbb{R}^{m}$ and $\alpha, \delta>0$, define the quantity

$$
P_{\delta}^{\alpha}(A):=\sup \sum_{i}\left(\operatorname{diam} B_{i}\right)^{\alpha},
$$

where the supremum is taken over all collections $\left\{B_{i}\right\}$ of pairwise disjoint closed balls centered at points of the set $A$ with diameters not exceeding $\delta$. The $\alpha$-dimensional packing premeasure of $A$ is defined as

$$
P^{\alpha}(A):=\lim _{\delta \rightarrow 0} P_{\delta}^{\alpha}(A)=\inf _{\delta>0} P_{\delta}^{\alpha}(A) .
$$

Using method I one can obtain an outer measure from $P^{\alpha}$ by letting

$$
\mathcal{P}^{\alpha}(A):=\inf \left\{\sum_{i} P^{\alpha}\left(A_{i}\right): A \subset \bigcup_{i} A_{i}\right\} .
$$

The set function $\mathcal{P}^{\alpha}$ is known as the packing measure. It is a Borel regular measure.

The quantity $\underline{g}_{\infty, \alpha}(A)^{\alpha}$ is known as the $\alpha$-dimensional Hewitt-Stromberg content. The $\alpha$-dimensional Hewitt-Stromberg measure is also constructed using method I [24, Exercise 10.51]:

$$
\mu^{\alpha}(A)=\inf \left\{\sum_{i} \underline{g}_{\infty, \alpha}\left(A_{i}\right)^{\alpha}: A \subset \bigcup_{i} A_{i}\right\} .
$$

Its properties were first studied in [19]. Some applications of the HewittStromberg measure were considered in $[\mathbf{4 4}]$. 
Our result. It was proved in [14] that for every compact set in $A \subset$ $\mathbb{R}^{m}$ such that $P^{\alpha}(A)<\infty$, there holds $\mathcal{P}^{\alpha}(A)=P^{\alpha}(A)$. Below, we obtain an analogue of this result for the outer measures constructed from premeasures $\underline{g}_{s, \alpha}(A)^{-\alpha / s}$ and $\bar{g}_{\infty, \alpha}(A)^{\alpha}$ using method I.

Theorem 3. Let $m \in \mathbb{N}$ and $0<\alpha \leq m$. Then for every compact set $A \subset \mathbb{R}^{m}$ with $P^{\alpha}(A)<\infty$, we have

$$
\underline{g}_{s, \alpha}(A)=\nu_{s, \alpha}(A)^{-s / \alpha}, \quad \alpha<s<\infty,
$$

and

$$
\bar{g}_{\infty, \alpha}(A)=\nu_{\infty, \alpha}(A)^{1 / \alpha} .
$$

Known results for compact $d$-rectifiable sets in $\mathbb{R}^{m}(d \leq m)$ obtained in $[\mathbf{2 7}],[\mathbf{2 2}],[\mathbf{2 3}],[\mathbf{7}]$, and [5] express the normalized limits of the minimum Riesz energy and $\epsilon$-complexity via the $d$-dimensional Hausdorff measure. Our result implies that on the class of compact sets of finite $d$-dimensional packing premeasure the lower limit of the minimum Riesz energy coincides with a certain outer measure.

Remark 3. The assumption $P^{\alpha}(A)<\infty$ cannot be replaced with the assumption $\underline{g}_{s, \alpha}(A)^{-\alpha / s}<\infty$. As a counterexample one can take the set $A_{q}=\left\{\frac{1}{n^{q}}: n \in \mathbb{N}\right\} \cup\{0\}, q>0$, and $\alpha \in(0,1)$. Since the Minkowski content of dimension $1 /(q+1)$ of the set $A_{q}$ is finite and positive, we, in particular, have $\underline{g}_{s, \alpha}(A)^{-\alpha / s}>0$ and $\bar{g}_{\infty, \alpha}(A)^{\alpha}>0$. On the other hand,

$$
\nu_{s, \alpha}(A) \leq \sum_{n=1}^{\infty} \underline{g}_{s, \alpha}(\{1 / n\})^{-\alpha / s}+\underline{g}_{s, \alpha}(\{0\})^{-\alpha / s}=0
$$

and

$$
\nu_{\infty, \alpha}(A) \leq \sum_{n=1}^{\infty} \bar{g}_{\infty, \alpha}(\{1 / n\})^{\alpha}+\bar{g}_{\infty, \alpha}(\{0\})^{\alpha}=0,
$$

which contradicts to the equalities asserted by Theorem 3 .

Remark 4. If one applies method I to the set function (premeasure) $\tau(A)=\bar{g}_{s, \alpha}(A)^{-\alpha / s}$, then for the resulting outer measure

$$
\tau_{s, \alpha}(A)=\inf \left\{\sum_{i} \bar{g}_{s, \alpha}\left(A_{i}\right)^{-\alpha / s}: A \subset \bigcup_{i} A_{i}\right\}, \quad s>\alpha,
$$

all Borel sets will be measurable. Indeed, the set function $\bar{g}_{s, \alpha}(\cdot)^{-\alpha / s}$ is monotone and supadditive on metrically separated sets, i.e. for every sets $A, B \subset \mathbb{R}^{m}$ such that $\operatorname{dist}(A, B)>0$, we have (see [23, Lemma 3.3])

$$
\bar{g}_{s, \alpha}(A \cup B)^{-\alpha / s} \geq \bar{g}_{s, \alpha}(A)^{-\alpha / s}+\bar{g}_{s, \alpha}(B)^{-\alpha / s}, \quad s>\alpha .
$$


By the general result of Haase [20, Theorem 2], the outer measure $\tau_{s, \alpha}$ coincides with the method II outer measure constructed from the premeasure $\bar{g}_{s, \alpha}(\cdot)^{-\alpha / s}$. It is a metric outer measure, which implies the measurability of all Borel sets.

\section{Auxiliary statements}

The following regularity result for the packing premeasure was proved in [14, Lemma 2.1].

Lemma 1. Let $m \in \mathbb{N}, 0<\alpha \leq m$, and $K$ be a compact set in $\mathbb{R}^{m}$ with $P^{\alpha}(K)<\infty$. Then for every subset $F \subset K$ and every $\epsilon>0$, there exists an open set $U_{\epsilon} \subset \mathbb{R}^{m}$ containing $F$ such that

$$
P^{\alpha}\left(K \cap U_{\epsilon}\right)<P^{\alpha}(F)+\epsilon \text {. }
$$

Using Lemma 1 we prove an analogous regularity result for the limits involving the minimum Riesz energy. We recall that $\mathcal{E}_{s}(A, N)=$ $\mathcal{E}_{s}(\bar{A}, N)$.

Lemma 2. Let $m \in \mathbb{N}, 0<\alpha \leq m, s>\alpha$, and $K$ be a compact set in $\mathbb{R}^{m}$ with $P^{\alpha}(K)<\infty$. Then for every compact subset $F \subset K$,

$$
\underline{g}_{s, \alpha}(F)=\sup _{\substack{U \supset F \\ U \text {-open }}} \underline{g}_{s, \alpha}(K \cap U)
$$

and

$$
\bar{g}_{s, \alpha}(F)=\sup _{\substack{U \supset F \\ U \text {-open }}} \bar{g}_{s, \alpha}(K \cap U) .
$$

Lemma 2 is used in the proof of Theorems 2 and 3. We prove this lemma combining the ideas from $[\mathbf{1 4}]$ and $[\mathbf{5}]$. We first verify the following statement.

Proposition 1. Let $m \in \mathbb{N}, 0<\alpha \leq m, \epsilon>0, G \subset H \subset \mathbb{R}^{m}$ be two compact sets such that $P^{\alpha}(H)<P^{\alpha}(G)+\epsilon$ and $\delta>0$ be such that $P_{\delta}^{\alpha}(H) \leq P^{\alpha}(H)+\epsilon$. Let $X_{M}:=\left\{z_{1}, \ldots, z_{M}\right\}$ be a collection of points in $H$ such that $\left|z_{i}-z_{j}\right| \geq \delta, 1 \leq i \neq j \leq M$, and $Y_{M}=\left\{z \in X_{M}\right.$ : $\operatorname{dist}(z, G)<\delta\}$. Then $\#\left(X_{M} \backslash Y_{M}\right) \leq 3 \cdot 2^{\alpha} \epsilon \delta^{-\alpha}$.

Proof of Proposition 1: Let $\left\{D_{i}\right\}$ be a collection of pairwise disjoint closed balls centered at points of $G$ with diameters at most $\delta / 2$ such that

$$
\sum_{i}\left(\operatorname{diam} D_{i}\right)^{\alpha}>P_{\delta / 2}^{\alpha}(G)-\epsilon \geq P^{\alpha}(G)-\epsilon
$$


Let $B[a, r]$ denote the closed ball in $\mathbb{R}^{m}$ of radius $r$ centered at point $a$. For every $x \neq y \in X_{M} \backslash Y_{M}$, by assumption we have $|x-y| \geq \delta$ and

$$
B[x, \delta / 4] \cap B[y, \delta / 4]=\emptyset .
$$

Balls from collection $\left\{D_{i}\right\}$ (their centers are in $G$ and diameters are at most $\delta / 2$ ) do not intersect balls $B[x, \delta / 4], x \in X_{M} \backslash Y_{M}$, since their centers are at least $\delta$ from $G$. Hence, $\left\{D_{i}\right\} \cup\{B[x, \delta / 4]\}_{x \in X_{M} \backslash Y_{M}}$ is a collection of pairwise disjoint closed balls centered in $H$ with diameters at most $\delta / 2$ and we obtain

$$
\sum_{i}\left(\operatorname{diam} D_{i}\right)^{\alpha}+2^{-\alpha} \sum_{x \in X_{M} \backslash Y_{M}} \delta^{\alpha} \leq P_{\delta / 2}^{\alpha}(H) .
$$

Then taking into account (24) we get

$$
\begin{aligned}
2^{-\alpha} \delta^{\alpha} \#\left(X_{M} \backslash Y_{M}\right) & \leq P_{\delta}^{\alpha}(H)-\sum_{i}\left(\operatorname{diam} D_{i}\right)^{\alpha} \\
& \leq P^{\alpha}(H)+\epsilon-P^{\alpha}(G)+\epsilon<3 \epsilon .
\end{aligned}
$$

Hence, \# $\left(X_{M} \backslash Y_{M}\right) \leq 3 \cdot 2^{\alpha} \epsilon \delta^{-\alpha}$, which proves Proposition 1 .

For every $\epsilon \in\left(0, \frac{1}{4^{4 \alpha}}\right)$, denote by $U_{\epsilon}$ an open set in $\mathbb{R}^{m}$ containing the compact subset $F \subset K$ such that

$$
P^{\alpha}\left(K \cap U_{\epsilon}\right)<P^{\alpha}(F)+\epsilon
$$

(such a set $U_{\epsilon}$ exists in view of Lemma 1 ). Let $[t]$ denote the largest integer not exceeding $t$. The following statement is true.

Proposition 2. Let $C$ be a positive and finite number and $\epsilon \in\left(0, \frac{1}{4^{4 \alpha}}\right)$ be such that $\chi_{\epsilon}(C):=1-\epsilon^{\frac{s}{4 \alpha}}(C+1)-3 \epsilon^{1 / 2}>0$. If $\mathcal{N} \subset \mathbb{N}$ is an infinite subset such that the following limit

$$
\lim _{\mathcal{N} \ni N \rightarrow \infty} \frac{\mathcal{E}_{s}\left(\overline{K \cap U_{\epsilon}}, N\right)}{N^{1+s / \alpha}}
$$

exists and does not exceed $C$, then

$$
C \geq\left(1-4 \epsilon^{1 /(4 \alpha)}\right)^{s} \chi_{\epsilon}(C)^{1+s / \alpha} \limsup _{\mathcal{N} \ni N \rightarrow \infty} \frac{\mathcal{E}_{s}\left(F,\left[\chi_{\epsilon}(C) N\right]\right)}{\left[\chi_{\epsilon}(C) N\right]^{1+s / \alpha}} .
$$

Proof of Proposition 2: Let $\omega_{N}^{*}:=\left\{x_{1, N}, \ldots, x_{N, N}\right\}, N \in \mathcal{N}$, be a sequence of energy minimizing configurations on $\overline{K \cap U_{\epsilon}}$. Denote $h:=$ $\epsilon^{1 /(4 \alpha)}, r_{i, N}:=\min _{j: j \neq i}\left|x_{i, N}-x_{j, N}\right|, i=1, \ldots, N$, and let

$$
\omega_{N}^{1}:=\left\{x_{i, N} \in \omega_{N}^{*}: r_{i, N} \geq h N^{-1 / \alpha}\right\} .
$$


We next show that $\omega_{N}^{1}$ contains sufficiently many points. We have

$$
\begin{aligned}
\mathcal{E}_{s}\left(\overline{K \cap U_{\epsilon}}, N\right) & =\sum_{i=1}^{N} \sum_{\substack{j=1 \\
j \neq i}}^{N} \frac{1}{\left|x_{i, N}-x_{j, N}\right|^{s}} \geq \sum_{i=1}^{N} \frac{1}{r_{i, N}^{s}} \geq \sum_{x_{i, N} \in \omega_{N}^{*} \backslash \omega_{N}^{1}} \frac{1}{r_{i, N}^{s}} \\
& \geq \sum_{x_{i, N} \in \omega_{N}^{*} \backslash \omega_{N}^{1}} h^{-s} N^{s / \alpha}=\#\left(\omega_{N}^{*} \backslash \omega_{N}^{1}\right) h^{-s} N^{s / \alpha} .
\end{aligned}
$$

By assumption, for $N \in \mathcal{N}$ sufficiently large, we have

$$
\mathcal{E}_{s}\left(\overline{K \cap U_{\epsilon}}, N\right) \leq(C+1) N^{1+s / \alpha} .
$$

Hence, $\#\left(\omega_{N}^{*} \backslash \omega_{N}^{1}\right) \leq h^{s}(C+1) N$ and

$$
\# \omega_{N}^{1} \geq\left(1-h^{s}(C+1)\right) N \text {. }
$$

Let now

$$
\omega_{N}^{2}:=\left\{x \in \omega_{N}^{1}: \operatorname{dist}(x, F)<2 h^{2} N^{-1 / \alpha}\right\} .
$$

We next show that the collection $\omega_{N}^{2}$ contains sufficiently many elements. Applying Proposition 1 with $G=F, H=\overline{K \cap U_{\epsilon}}, X_{M}=\omega_{N}^{1}, Y_{M}=\omega_{N}^{2}$, $\delta=2 h^{2} N^{-1 / \alpha}$, and taking into account that $h \in\left(0, \frac{1}{4}\right)$, for $N \in \mathcal{N}$ sufficiently large, we get that

$$
\#\left(\omega_{N}^{1} \backslash \omega_{N}^{2}\right) \leq 3 \epsilon h^{-2 \alpha} N=3 \epsilon^{1 / 2} N
$$

and hence,

$$
\# \omega_{N}^{2} \geq\left(1-h^{s}(C+1)-3 \epsilon^{1 / 2}\right) N=\chi_{\epsilon}(C) N .
$$

For every $x \in \omega_{N}^{2}$ let $y_{x}$ be an element in $F$ such that $\operatorname{dist}\left(x, y_{x}\right)<$ $2 h^{2} N^{-1 / \alpha}$. Denote $\omega_{F}:=\left\{y_{x}: x \in \omega_{N}^{2}\right\}$. Note that for every $x \neq z \in$ $\omega_{N}^{2}$, we have $|x-z| \geq h N^{-1 / \alpha}$ and

$$
\begin{aligned}
\left|y_{x}-y_{z}\right| & \geq|x-z|-\left|x-y_{x}\right|-\left|z-y_{z}\right| \\
& \geq|x-z|-4 h^{2} N^{-1 / \alpha} \geq(1-4 h)|x-z|>0,
\end{aligned}
$$

since $h \in(0,1 / 4)$. Then we have $\# \omega_{F}=\# \omega_{N}^{2}$. By the assumptions of the lemma, taking into account (26), we obtain

$$
\begin{aligned}
C & \geq \lim _{\mathcal{N} \ni N \rightarrow \infty} \frac{E_{s}\left(\omega_{N}^{*}\right)}{N^{1+s / \alpha}} \geq \limsup _{\mathcal{N} \ni N \rightarrow \infty} \frac{E_{s}\left(\omega_{N}^{2}\right)}{N^{1+s / \alpha}} \geq(1-4 h)^{s} \limsup _{\mathcal{N} \ni N \rightarrow \infty} \frac{E_{s}\left(\omega_{F}\right)}{N^{1+s / \alpha}} \\
& \geq(1-4 h)^{s} \chi_{\epsilon}(C)^{1+s / \alpha} \limsup _{\mathcal{N} \ni N \rightarrow \infty} \frac{\mathcal{E}_{s}\left(F,\left[\chi_{\epsilon}(C) N\right]\right)}{\left[\chi_{\epsilon}(C) N\right]^{1+s / \alpha}},
\end{aligned}
$$

which completes the proof of Proposition 2 . 
Proof of Lemma 2: Denote

$$
C_{0}:=\sup _{\substack{U \supset F \\ U \text {-open }}} \underline{g}_{s, \alpha}(K \cap U) .
$$

By monotonicity of the Riesz $s$-energy, $C_{0} \leq \underline{g}_{s, \alpha}(F)$. If $C_{0}=\infty$, then $\underline{g}_{s, \alpha}(F)=\infty$ and (22) holds trivially. Assume that $C_{0}<\infty$. For every $\epsilon \in\left(0,4^{-4 \alpha}\right)$ such that $\chi_{\epsilon}\left(C_{0}+\epsilon\right)>0$, let $\mathcal{N} \subset \mathbb{N}$ be an infinite subsequence such that

$$
\underline{g}_{s, \alpha}\left(\overline{K \cap U_{\epsilon}}\right)=\lim _{\mathcal{N} \ni N \rightarrow \infty} \frac{\mathcal{E}_{s}\left(\overline{K \cap U_{\epsilon}}, N\right)}{N^{1+s / \alpha}}<C_{0}+\epsilon .
$$

Using Proposition 2 we obtain

$$
\begin{aligned}
C_{0}+\epsilon & \geq\left(1-4 \epsilon^{1 /(4 \alpha)}\right)^{s} \chi_{\epsilon}\left(C_{0}+\epsilon\right)^{1+s / \alpha} \limsup _{\mathcal{N} \ni N \rightarrow \infty} \frac{\mathcal{E}_{s}\left(F,\left[\chi_{\epsilon}\left(C_{0}+\epsilon\right) N\right]\right)}{\left[\chi_{\epsilon}\left(C_{0}+\epsilon\right) N\right]^{1+s / \alpha}} \\
& \geq\left(1-4 \epsilon^{1 /(4 \alpha)}\right)^{s} \chi_{\epsilon}\left(C_{0}+\epsilon\right)^{1+s / \alpha} \underline{g}_{s, \alpha}(F) .
\end{aligned}
$$

Since $C_{0}$ does not depend on $\epsilon$, we have $\chi_{\epsilon}\left(C_{0}+\epsilon\right) \rightarrow 1, \epsilon \rightarrow 0$. Hence, $C_{0} \geq \underline{g}_{s, \alpha}(F)$ and we get equality $(22)$.

Analogously, we now let

$$
C_{1}:=\sup _{\substack{U \supset F \\ U \text {-open }}} \bar{g}_{s, \alpha}(K \cap U) .
$$

We have again, $C_{1} \leq \bar{g}_{s, \alpha}(F)$ and it remains to consider the case $C_{1}<\infty$. Let $\mathcal{N}_{1} \subset \mathbb{N}$ be an infinite subset such that

$$
\bar{g}_{s, \alpha}(F)=\lim _{\mathcal{N}_{1} \ni N \rightarrow \infty} \frac{\mathcal{E}_{s}(F, N)}{N^{1+s / \alpha}} .
$$

Let $\epsilon \in\left(0,4^{-4 \alpha}\right)$ be sufficiently small so that $0<\chi_{\epsilon}\left(C_{1}+\epsilon\right)<1$. Then for every $M \in \mathcal{N}_{1}$, there is a number $N_{M} \in \mathbb{N}$ such that $\left[\chi_{\epsilon}\left(C_{1}+\epsilon\right) N_{M}\right]=$ $M$. Let $\mathcal{N}_{2}=\left\{N_{M}\right\}_{M \in \mathcal{N}_{1}}$. All partial limits of the sequence

$$
\frac{\mathcal{E}_{s}\left(\overline{K \cap U_{\epsilon}}, N\right)}{N^{1+s / \alpha}}, \quad N \in \mathcal{N}_{2},
$$

belong to the interval $\left[0, C_{1}\right]$. Denote by $\mathcal{N}$ an infinite subset of $\mathcal{N}_{2}$ such that the limit

$$
\lim _{\mathcal{N} \ni N \rightarrow \infty} \frac{\mathcal{E}_{s}\left(\overline{K \cap U_{\epsilon}}, N\right)}{N^{1+s / \alpha}}
$$


exists. It will not exceed $C_{1}+\epsilon$. By Proposition 2, we have

$$
\begin{aligned}
C_{1}+\epsilon & \geq\left(1-4 \epsilon^{1 /(4 \alpha)}\right)^{s} \chi_{\epsilon}\left(C_{1}+\epsilon\right)^{1+s / \alpha} \limsup _{\mathcal{N} \ni N \rightarrow \infty} \frac{\mathcal{E}_{s}\left(F,\left[\chi_{\epsilon}\left(C_{1}+\epsilon\right) N\right]\right)}{\left[\chi_{\epsilon}\left(C_{1}+\epsilon\right) N\right]^{1+s / \alpha}} \\
& =\left(1-4 \epsilon^{1 /(4 \alpha)}\right)^{s} \chi_{\epsilon}\left(C_{1}+\epsilon\right)^{1+s / \alpha} \lim _{\mathcal{N}_{1} \ni N \rightarrow \infty} \frac{\mathcal{E}_{s}(F, N)}{N^{1+s / \alpha}} \\
& =\left(1-4 \epsilon^{1 /(4 \alpha)}\right)^{s} \chi_{\epsilon}\left(C_{1}+\epsilon\right)^{1+s / \alpha} \bar{g}_{s, \alpha}(F) .
\end{aligned}
$$

Letting $\epsilon \rightarrow 0$, we get that $\chi_{\epsilon}\left(C_{1}+\epsilon\right) \rightarrow 1$. Hence, $C_{1} \geq \bar{g}_{s, \alpha}(F)$ and we get equality (23). Lemma 2 is proved.

We will also need the following statement, see [23, Lemma 3.2]. The assumption of boundedness of $B$ and $D$ as well as the assumption that $\lambda$ is an integer made in $[\mathbf{2 3}]$ are not essential.

Lemma 3. Let $m \in \mathbb{N}, 0<\lambda \leq m, s>\lambda$ and $B$ and $D$ be arbitrary sets in $\mathbb{R}^{m}$. Then

$$
\underline{g}_{s, \lambda}(B \cup D)^{-\lambda / s} \leq \underline{g}_{s, \lambda}(B)^{-\lambda / s}+\underline{g}_{s, \lambda}(D)^{-\lambda / s} .
$$

Furthermore, if $\underline{g}_{s, \lambda}(B), \underline{g}_{s, \lambda}(D)>0$ and at least one of these quantities is finite, then for any infinite subset $\mathcal{N} \subset \mathbb{N}$ and sequence $\left\{\omega_{N}\right\}_{N \in \mathcal{N}}$ of $N$-point configurations in $B \cup D$ such that

$$
\lim _{\mathcal{N} \ni N \rightarrow \infty} \frac{E_{s}\left(\omega_{N}\right)}{N^{1+s / \lambda}}=\left(\underline{g}_{s, \lambda}(B)^{-\lambda / s}+\underline{g}_{s, \lambda}(D)^{-\lambda / s}\right)^{-s / \lambda}
$$

holds, we have

$$
\lim _{\mathcal{N} \ni N \rightarrow \infty} \frac{\#\left(\omega_{N} \cap B\right)}{N}=\frac{\underline{g}_{s, \lambda}(D)^{\lambda / s}}{\underline{g}_{s, \lambda}(B)^{\lambda / s}+\underline{g}_{s, \lambda}(D)^{\lambda / s}} .
$$

In the case $\underline{g}_{s, \lambda}(D)=\infty$ the right-hand side of relation (27) is understood to be 1 and we agree that $\infty^{-\lambda / s}=0$ and $0^{-s / \lambda}=\infty$.

\section{Proof of Theorem 2}

We start by verifying the following basic statement.

Proposition 3. Let $K \subset \mathbb{R}^{m}$ be a self-similar set satisfying the OSC and having Hausdorff dimension $\lambda$. Then for every $s>\lambda$,

$$
0<\underline{g}_{s, \lambda}(K) \leq \bar{g}_{s, \lambda}(K)<\infty .
$$


Proof of Proposition 3: Let $\left\{x_{1, N}, \ldots, x_{N, N}\right\}, N \in \mathbb{N}, N \geq 2$, be an $s$-energy minimizing configuration on $K$. Denote

$$
r_{i, N}=\frac{1}{2} \min _{j: j \neq i}\left|x_{i, N}-x_{j, N}\right|, \quad i=1, \ldots, N .
$$

Since $K$ is compact, the number $L_{N}$ of indices $i$, for which $r_{i, N}>1$, stays bounded. Using the convexity of the function $y(t)=t^{-s / \lambda}$, we have

$$
\begin{aligned}
\mathcal{E}_{s}(K, N) & =\sum_{i=1}^{N} \sum_{\substack{j=1 \\
j \neq i}}^{N} \frac{1}{\left|x_{i, N}-x_{j, N}\right|^{s}} \geq \sum_{i=1}^{N} \frac{1}{\left(2 r_{i, N}\right)^{s}} \geq \sum_{i: r_{i, N} \leq 1} \frac{1}{\left(2 r_{i, N}\right)^{s}} \\
& =2^{-s}\left(N-L_{N}\right) \cdot \frac{1}{N-L_{N}} \cdot \sum_{i: r_{i, N} \leq 1}\left(r_{i, N}^{\lambda}\right)^{-s / \lambda} \\
& \geq 2^{-s}\left(N-L_{N}\right)^{1+s / \lambda}\left(\sum_{i: r_{i, N} \leq 1} r_{i, N}^{\lambda}\right)^{-s / \lambda} .
\end{aligned}
$$

By Theorem 1, there is a number $a>0$ such that

$$
\begin{aligned}
\liminf _{N \rightarrow \infty} \frac{\mathcal{E}_{s}(K, N)}{N^{1+s / \lambda}} & \geq 2^{-s} \liminf _{N \rightarrow \infty}\left(\frac{1}{a} \sum_{i: r_{i, N} \leq 1} \mathcal{H}_{\lambda}\left(K \cap B\left(x_{i, N}, r_{i, N}\right)\right)\right)^{-s / \lambda} \\
& =2^{-s} a^{s / \lambda} \liminf _{N \rightarrow \infty}\left(\mathcal{H}_{\lambda}\left(K \cap\left(\cup_{i: r_{i, N} \leq 1} B\left(x_{i, N}, r_{i, N}\right)\right)\right)\right)^{-s / \lambda} \\
& \geq 2^{-s} a^{s / \lambda} \mathcal{H}_{\lambda}(K)^{-s / \lambda} .
\end{aligned}
$$

Since by Theorem $1,0<\mathcal{H}_{\lambda}(K)<\infty$, we get that

$$
\underline{g}_{s, \lambda}(K) \geq 2^{-s} a^{s / \lambda} \mathcal{H}_{\lambda}(K)^{-s / \lambda}>0, \quad s>\lambda .
$$

Corollary 1 of Theorem 4 in [5] implies that whenever compact set $K$ has positive $\mathcal{H}_{\lambda}$-measure, there exists a finite and positive constant $C=$ $C(K, \lambda, s)$ such that for every $N$ sufficiently large,

$$
\mathcal{E}_{s}(K, N) \leq C N^{1+s / \lambda}, \quad s>\lambda .
$$

Hence, $\bar{g}_{s, \lambda}(K) \leq C<\infty$. Proposition 3 is proved.

For every positive integer $n$ and every vector of indexes $\bar{i}=\left\{i_{1}, \ldots, i_{n}\right\}$, $i_{j}=1, \ldots, p, j=1, \ldots, n$, let

$$
K_{\bar{i}}=S_{i_{1}} \circ \cdots \circ S_{i_{n}}(K) .
$$


Denote by $\mathcal{F}_{n}(K)$ the family of all sets $K_{\bar{i}}$, where $\bar{i} \in\{1, \ldots, p\}^{n}$. It is not difficult to see that for every $n \in \mathbb{N}$,

$$
K=\bigcup_{\bar{i} \in\{1, \ldots, p\}^{n}} K_{\bar{i}} .
$$

Denote also by $\mathcal{C}(K)$ the family of all non-empty compact subsets of the set $K$. Our proof relies on the following statement, which could be considered as an analogue of the result in [25] about the unicity of the invariant measure.

Lemma 4. Let $K \subset \mathbb{R}^{m}$ be a self-similar set such that $0<\mathcal{H}_{\lambda}(K)<$ $\infty$. There exists a unique non-negative functional $\psi$ defined on $\mathcal{C}(K)$ satisfying the following properties:

(1) For any compact subsets $A \subset B \subset K$, there holds $\psi(A) \leq \psi(B)$.

(2) For every $n \in \mathbb{N}$ and $\bar{i}=\left\{i_{1}, \ldots, i_{n}\right\} \in\{1, \ldots, p\}^{n}$, there holds

$$
\psi\left(K_{\bar{i}}\right)=\sigma_{i_{1}}^{\lambda} \cdot \ldots \cdot \sigma_{i_{n}}^{\lambda} .
$$

(3) For any $n \in \mathbb{N}$ and any collection of pairwise distinct sets $F_{1}, \ldots$, $F_{k} \in \mathcal{F}_{n}(K)$, there holds

$$
\psi\left(\bigcup_{i=1}^{k} F_{i}\right)=\sum_{i=1}^{k} \psi\left(F_{i}\right) .
$$

(4) For every $A \in \mathcal{C}(K)$ and every $\epsilon>0$, there is an open set $U$ such that $A \subset U$ and

$$
\psi(\overline{U \cap K}) \leq \psi(A)+\epsilon .
$$

Note that the assumptions of this lemma imply that $\psi(K)=1$.

Proof of Lemma 4: The existence follows from the fact that the functional $\psi$ such that

$$
\psi(A)=h_{\lambda, K}(A), \quad A \in \mathcal{C}(K),
$$

satisfies properties (1)-(4).

Show uniqueness. Let $\psi_{1}$ and $\psi_{2}$ be arbitrary functionals satisfying properties (1)-(4). Choose any set $A \in \mathcal{C}(K)$. For every $\epsilon>0$, let $U$ be an open set containing $A$ such that

$$
\psi_{2}(\overline{K \cap U}) \leq \psi_{2}(A)+\epsilon .
$$

Then, there exists a $\delta$-neighborhood $U_{\delta}$ of the set $A$, so that $U_{\delta} \cap K \subset$ $K \cap U$ and

$$
\psi_{2}\left(\overline{K \cap U_{\delta}}\right) \leq \psi_{2}(A)+\epsilon .
$$


Let $S_{1}, \ldots, S_{p}$ be the contracting similitudes defining the set $K$ and $\sigma_{1}, \ldots, \sigma_{p}$ be their contraction coefficients. Denote $\sigma=\max \left\{\sigma_{1}, \ldots, \sigma_{p}\right\}$. Choose $n$ to be a sufficiently large positive integer so that $\sigma^{n} \cdot \operatorname{diam} K<\delta$. Denote by $Z$ the set of vector indices $\bar{i}=\left(i_{1}, \ldots, i_{n}\right) \in\{1, \ldots, p\}^{n}$ such that

$$
A \cap K_{\bar{i}} \neq \emptyset .
$$

Then

$$
A \subset \bigcup_{\bar{i} \in Z} K_{\bar{i}} \subset U_{\delta} \cap K .
$$

Taking into account (28), we get

$$
\begin{aligned}
\psi_{1}(A) & \leq \psi_{1}\left(\bigcup_{\bar{i} \in Z} K_{\bar{i}}\right)=\sum_{\bar{i} \in Z} \psi_{1}\left(K_{\bar{i}}\right)=\sum_{\bar{i} \in Z} \sigma_{i_{1}}^{\lambda} \cdot \ldots \cdot \sigma_{i_{n}}^{\lambda} \\
& =\sum_{\bar{i} \in Z} \psi_{2}\left(K_{\bar{i}}\right)=\psi_{2}\left(\bigcup_{\bar{i} \in Z} K_{\bar{i}}\right) \leq \psi_{2}\left(\overline{U_{\delta} \cap K}\right) \leq \psi_{2}(A)+\epsilon .
\end{aligned}
$$

Letting $\epsilon \rightarrow 0$, we get $\psi_{1}(A) \leq \psi_{2}(A)$. Since $\psi_{1}$ and $\psi_{2}$ were chosen arbitrarily, we can do the same proof to show the opposite inequality. Lemma 4 is proved.

We now proceed with the proof of Theorem 2. For $s>\lambda$, let us show that the following functional

$$
\psi(A)=\left(\frac{\underline{g}_{s, \lambda}(A)}{\underline{g}_{s, \lambda}(K)}\right)^{-\lambda / s}
$$

satisfies properties (1)-(4) in Lemma 4. In view of Proposition 3, the functional $\psi$ is well defined. Whenever $A \subset B \subset \mathbb{R}^{m}$, we have

$$
\mathcal{E}_{s}(A, N) \geq \mathcal{E}_{s}(B, N), \quad N \geq 2,
$$

which implies property (1). If $S: \mathbb{R}^{m} \rightarrow \mathbb{R}^{m}$ is a similitude with the contraction coefficient $\sigma$, then for every set $A \subset \mathbb{R}^{m}$,

$$
\mathcal{E}_{s}(S(A), N)=\sigma^{-s} \mathcal{E}_{s}(A, N), \quad N \geq 2 .
$$

This implies property (2). Lemma 3 shows that $\psi$ is a finitely subadditive set function. If now, $H$ is any collection of pairwise distinct sets 
from $\mathcal{F}_{n}(K)$ and $G$ is the collection of the remaining sets from $\mathcal{F}_{n}(K)$, then taking into account property (2) and relation (11), we obtain

$$
\begin{aligned}
1 & =\psi(K) \leq \psi\left(\bigcup_{B \in H} B\right)+\psi\left(\bigcup_{B \in G} B\right) \leq \sum_{B \in H} \psi(B)+\sum_{B \in G} \psi(B) \\
& =\sum_{B \in \mathcal{F}_{n}(K)} \psi(B)=\sum_{i_{1}=1}^{p} \cdots \sum_{i_{n}=1}^{p} \sigma_{i_{1}}^{\lambda} \cdot \cdots \cdot \sigma_{i_{n}}^{\lambda}=1 .
\end{aligned}
$$

Hence, the equality sign must be everywhere in the above relation and we get that

$$
\psi\left(\bigcup_{B \in H} B\right)=\sum_{B \in H} \psi(B)
$$

which proves property (3). Relation (12) from Theorem 1 implies that $P^{\lambda}(K)<\infty$ and property (4) for the functional $\psi$ will follow from relation (22) in Lemma 2 . Since $h_{\lambda, K}$ also satisfies properties (1)-(4), in view of the uniqueness, we get that

$$
\psi(A)=\left(\frac{\underline{g}_{s, \lambda}(A)}{\underline{g}_{s, \lambda}(K)}\right)^{-\lambda / s}=h_{\lambda, K}(A), \quad A \in \mathcal{C}(K),
$$

and relation (14) will follow.

To prove relation (15) we will need a stronger statement than relation (21) for compact subsets of a self-similar set.

Lemma 5. Let $K$ be a self-similar set of Hausdorff dimension $\lambda$ satisfying the $O S C$ and $s>\lambda$. Then for every compact subsets $B$ and $D$ of $K$ such that $\mathcal{H}_{\lambda}(B \cap D)=0$, there holds

$$
\bar{g}_{s, \lambda}(B \cup D)^{-\lambda / s} \geq \bar{g}_{s, \lambda}(B)^{-\lambda / s}+\bar{g}_{s, \lambda}(D)^{-\lambda / s} .
$$

Proof of Lemma 5: If $\bar{g}_{s, \lambda}(B)=\infty$ or $\bar{g}_{s, \lambda}(D)=\infty$, then the assertion of Lemma 5 holds trivially. Assume that $\bar{g}_{s, \lambda}(B), \bar{g}_{s, \lambda}(D)<\infty$. If $B \cap D=\emptyset$, then the assertion of Lemma 5 follows from (21). Assume that $B \cap D \neq \emptyset$. From relation (14), since $h_{\lambda, K}(B \cap D)=0$, we get $\underline{g}_{s, \lambda}(B \cap D)=\infty$. Then from Lemma 2 it follows that

$$
\sup _{\substack{U \supset B \cap D \\ U \text {-open }}} \underline{g}_{s, \lambda}(U \cap K)=\infty .
$$

For every $k \in \mathbb{N}$, let $U_{k}$ be an open set containing $B \cap D$ such that

$$
\underline{g}_{s, \lambda}\left(U_{k} \cap K\right)>k .
$$


Since $\operatorname{dist}\left(B \backslash U_{k}, D \backslash U_{k}\right)>0$, by relation (21) we have

$$
\begin{aligned}
\bar{g}_{s, \lambda}(B \cup D)^{-\lambda / s} & \geq \bar{g}_{s, \lambda}\left(\left(B \backslash U_{k}\right) \cup\left(D \backslash U_{k}\right)\right)^{-\lambda / s} \\
& \geq \bar{g}_{s, \lambda}\left(B \backslash U_{k}\right)^{-\lambda / s}+\bar{g}_{s, \lambda}\left(D \backslash U_{k}\right)^{-\lambda / s} .
\end{aligned}
$$

Denote by $\widetilde{\omega}_{N}, N \in \mathbb{N}, N \geq 2$, a sequence of $s$-energy minimizing configurations on $B$ such that $\# \widetilde{\omega}_{N}=N, N \geq 2$. Let also $a_{N}=$ $\#\left(\widetilde{\omega}_{N} \cap U_{k}\right)$,

$$
\beta_{k}=\limsup _{N \rightarrow \infty} \frac{a_{N}}{N},
$$

and $\mathcal{N} \subset \mathbb{N}$ be an infinite sequence such that

$$
\lim _{\mathcal{N} \ni N \rightarrow \infty} \frac{a_{N}}{N}=\beta_{k}
$$

If $\beta_{k}>0$, then

$\mathcal{E}_{s}(B, N)=E_{s}\left(\widetilde{\omega}_{N}\right) \geq E_{s}\left(\widetilde{\omega}_{N} \cap U_{k}\right) \geq \mathcal{E}_{s}\left(B \cap U_{k}, a_{N}\right) \geq \mathcal{E}_{s}\left(K \cap U_{k}, a_{N}\right)$,

and

$$
\begin{aligned}
\bar{g}_{s, \lambda}(B) & \geq \limsup _{\mathcal{N} \ni N \rightarrow \infty} \frac{\mathcal{E}_{s}(B, N)}{N^{1+s / \lambda}} \geq \limsup _{\mathcal{N} \ni N \rightarrow \infty} \frac{\mathcal{E}_{s}\left(K \cap U_{k}, a_{N}\right)}{a_{N}^{1+s / \lambda}} \cdot\left(\frac{a_{N}}{N}\right)^{1+s / \lambda} \\
& \geq \underline{g}_{s, \lambda}\left(U_{k} \cap K\right) \beta_{k}^{1+s / \lambda}>k \beta_{k}^{1+s / \lambda} .
\end{aligned}
$$

Hence, $\beta_{k}<\left(\bar{g}_{s, \lambda}(B) / k\right)^{1 /(1+s / \lambda)}$ including the case $\beta_{k}=0$. Assume $k$ to be sufficiently large so that $\gamma_{k}=1-\beta_{k}-\frac{1}{k}>0$ and let $b_{N}=$ $\#\left(\widetilde{\omega}_{N} \backslash U_{k}\right), N \in \mathbb{N}, N \geq 2$. Then $b_{N} \geq \gamma_{k} N$ for $N$ sufficiently large, and we obtain

$$
\begin{aligned}
\bar{g}_{s, \lambda}(B) & =\limsup _{N \rightarrow \infty} \frac{E_{s}\left(\widetilde{\omega}_{N}\right)}{N^{1+s / \lambda}} \geq \limsup _{N \rightarrow \infty} \frac{E_{s}\left(\widetilde{\omega}_{N} \backslash U_{k}\right)}{N^{1+s / \lambda}} \\
& \geq \limsup _{N \rightarrow \infty} \frac{\mathcal{E}_{s}\left(B \backslash U_{k}, b_{N}\right)}{N^{1+s / \lambda}} \\
& \geq \limsup _{N \rightarrow \infty} \frac{\mathcal{E}_{s}\left(B \backslash U_{k},\left[\gamma_{k} N\right]\right)}{\left[\gamma_{k} N\right]^{1+s / \lambda}} \cdot\left(\frac{\left[\gamma_{k} N\right]}{N}\right)^{1+s / \lambda} \\
& \geq \bar{g}_{s, \lambda}\left(B \backslash U_{k}\right) \gamma_{k}^{1+s / \lambda} .
\end{aligned}
$$

Using analogous argument, one can show that there is a sequence $\theta_{k} \rightarrow 1$, $k \rightarrow \infty$, such that

$$
\bar{g}_{s, \lambda}(D) \geq \bar{g}_{s, \lambda}\left(D \backslash U_{k}\right) \theta_{k}^{1+s / \lambda} .
$$


Then from (30), taking into account (31) and (32) we get

$$
\bar{g}_{s, \lambda}(B \cup D)^{-\lambda / s} \geq \bar{g}_{s, \lambda}(B)^{-\lambda / s} \gamma_{k}^{1+\lambda / s}+\bar{g}_{s, \lambda}(D)^{-\lambda / s} \theta_{k}^{1+\lambda / s} .
$$

Since $\gamma_{k}, \theta_{k} \rightarrow 1$, letting $k \rightarrow \infty$ we obtain the assertion of Lemma 5 .

Let now

$$
\varphi(A)=\left(\frac{\bar{g}_{s, \lambda}(A)}{\bar{g}_{s, \lambda}(K)}\right)^{-\lambda / s}, \quad A \in \mathcal{C}(K) .
$$

Proposition 3 implies that the set function $\varphi$ is well-defined. Properties (1) and (2) are proved in the same way as they were proved for $\psi$. Property (4) follows from relation (23) of Lemma 2. By Lemma 5, if $H$ is any collection of pairwise distinct sets from $\mathcal{F}_{n}(K)$ and $G$ is the collection of the remaining sets from $\mathcal{F}_{n}(K)$, then the intersection of the unions of these two collections has $\mathcal{H}_{\lambda}$-measure zero, and we obtain

$$
\begin{aligned}
1 & =\varphi(K) \geq \varphi\left(\bigcup_{B \in H} B\right)+\varphi\left(\bigcup_{B \in G} B\right) \geq \sum_{B \in H} \varphi(B)+\sum_{B \in G} \varphi(B) \\
& =\sum_{B \in \mathcal{F}_{n}(K)} \varphi(B)=\sum_{i_{1}=1}^{p} \cdots \sum_{i_{n}=1}^{p} \sigma_{i_{1}}^{\lambda} \cdot \ldots \cdot \sigma_{i_{n}}^{\lambda}=1 .
\end{aligned}
$$

Hence, the equality sign must be everywhere in the above relation and we get that

$$
\varphi\left(\bigcup_{B \in H} B\right)=\sum_{B \in H} \varphi(B)
$$

which proves property (3). Then, by Lemma 4 , we will have

$$
\varphi(A)=\left(\frac{\bar{g}_{s, \lambda}(A)}{\bar{g}_{s, \lambda}(K)}\right)^{-\lambda / s}=h_{\lambda, K}(A), \quad A \in \mathcal{C}(K),
$$

which will prove (15).

To prove relations (16) and (17) consider an arbitrary infinite subset $\mathcal{N} \in \mathbb{N}$ and an arbitrary sequence $\left\{\bar{\omega}_{N}\right\}_{N \in \mathcal{N}}$ of $N$-point configurations on $A$ such that

$$
\lim _{\mathcal{N} \ni N \rightarrow \infty} \frac{E_{s}\left(\bar{\omega}_{N}\right)}{N^{1+s / \lambda}}=\underline{g}_{s, \lambda}(A) .
$$

In the case $\underline{g}_{s, \lambda}(K)=\bar{g}_{s, \lambda}(K)$, in view of (14) and (15), the limit $g_{s, \lambda}(A)$ exists and any asymptotically energy minimizing sequence $\left\{\bar{\omega}_{N}\right\}_{N \in \mathbb{N}}$ will satisfy (33) with $\mathcal{N}=\mathbb{N}$. Therefore, we let $\mathcal{N}=\mathbb{N}$ in this case. 
Let $B \subset A$ be any non-empty subset such that the boundary $\partial_{A} B$ of $B$ relative to $A$ has $h_{\lambda, A}$-measure zero. Then $h_{\lambda, K}\left(\partial_{A} B\right)=0$ and in view of (14), we obtain

$$
\begin{aligned}
\lim _{\mathcal{N} \ni N \rightarrow \infty} \frac{E_{s}\left(\bar{\omega}_{N}\right)}{N^{1+s / \lambda}} & =\underline{g}_{s, \lambda}(A)=\underline{g}_{s, \lambda}(K)\left(h_{\lambda, K}(B)+h_{\lambda,}(A \backslash B)\right)^{-s / \lambda} \\
& =\underline{g}_{s, \lambda}(K)\left(h_{\lambda, K}(\bar{B})+h_{\lambda, K}(\overline{A \backslash B})\right)^{-s / \lambda} \\
& =\left(\underline{g}_{s, \lambda}(\bar{B})^{-\lambda / s}+\underline{g}_{s, \lambda}(\overline{A \backslash B})^{-\lambda / s}\right)^{-s / \lambda} \\
& =\left(\underline{g}_{s, \lambda}(B)^{-\lambda / s}+\underline{g}_{s, \lambda}(A \backslash B)^{-\lambda / s}\right)^{-s / \lambda}
\end{aligned}
$$

Since $h_{\lambda, K}(B), h_{\lambda, K}(A \backslash B)<\infty$ and at least one of these quantities is positive, by (14), we have $\underline{g}_{s, \lambda}(B), \underline{g}_{s, \lambda}(A \backslash B)>0$ and at least one of these quantities is finite. Then we can apply Lemma 3. From this lemma and relation (14) we obtain

$$
\lim _{\mathcal{N} \ni N \rightarrow \infty} \frac{\#\left(\bar{\omega}_{N} \cap B\right)}{N}=\frac{\underline{g}_{s, \lambda}(A \backslash B)^{\lambda / s}}{\underline{g}_{s, \lambda}(B)^{\lambda / s}+\underline{g}_{s, \lambda}(A \backslash B)^{\lambda / s}}=h_{\lambda, A}(B),
$$

which implies relations (16) and (17). Theorem 2 is proved.

Proof of Corollary 1: Relations (14) and (15) together with equalities (6) imply relations (18). The proof of relations (19) and (20) can be done analogously to the proof of relations (16) and (17). Let $\mathcal{N} \subset \mathbb{N}$ be arbitrary infinite subsequence and $\left\{\widetilde{\omega}_{N}\right\}_{N \in \mathcal{N}}$ be any sequence of $N$-point configurations on $A$ such that

$$
\lim _{\mathcal{N} \ni N \rightarrow \infty} \delta\left(\widetilde{\omega}_{N}\right) \cdot N^{1 / \lambda}=\bar{g}_{\infty, \lambda}(A) .
$$

In the case $\underline{g}_{\infty, \lambda}(K)=\bar{g}_{\infty, \lambda}(K)$, in view of (18), the limit $g_{\infty, \lambda}(A)$ exists and any asymptotically best-packing sequence $\left\{\widetilde{\omega}_{N}\right\}_{N \in \mathbb{N}}$ will satisfy (34) with $\mathcal{N}=\mathbb{N}$. Therefore, we let $\mathcal{N}=\mathbb{N}$ in this case. 
Let $B \subset A$ be any subset such that $h_{\lambda, A}\left(\partial_{A} B\right)=0$. Then $h_{\lambda, K}\left(\partial_{A} B\right)=$ 0 and in view of (18), we will have

$$
\begin{aligned}
\lim _{\mathcal{N} \ni N \rightarrow \infty} \delta\left(\widetilde{\omega}_{N}\right) \cdot N^{1 / \lambda} & =\bar{g}_{\infty, \lambda}(A)=\bar{g}_{\infty, \lambda}(K) h_{\lambda, K}(A)^{1 / \lambda} \\
& =\bar{g}_{\infty, \lambda}(K)\left(h_{\lambda, K}(B)+h_{\lambda, K}(A \backslash B)\right)^{1 / \lambda} \\
& =\bar{g}_{\infty, \lambda}(K)\left(h_{\lambda, K}(\bar{B})+h_{\lambda, K}(\overline{A \backslash B})\right)^{1 / \lambda} \\
& =\left(\bar{g}_{\infty, \lambda}(K)^{\lambda} h_{\lambda, K}(\bar{B})+\bar{g}_{\infty, \lambda}(K)^{\lambda} h_{\lambda, K}(\overline{A \backslash B})\right)^{1 / \lambda} \\
& =\left(\bar{g}_{\infty, \lambda}(\bar{B})^{\lambda}+\bar{g}_{\infty, \lambda}(\overline{A \backslash B})^{\lambda}\right)^{1 / \lambda} \\
& =\left(\bar{g}_{\infty, \lambda}(B)^{\lambda}+\bar{g}_{\infty, \lambda}(A \backslash B)^{\lambda}\right)^{1 / \lambda} .
\end{aligned}
$$

This shows that the sequence $\left\{\widetilde{\omega}_{N}\right\}_{N \in \mathcal{N}}$ satisfies relation (16) in Lemma 3.1 from [6]. Applying relation (15) from that lemma, relation (18) proved above and the fact that $h_{\lambda, K}\left(\partial_{A} B\right)=0$ and $0<\bar{g}_{\infty, \lambda}(K)<\infty$, we will obtain

$$
\begin{aligned}
\lim _{\mathcal{N} \ni N \rightarrow \infty} \frac{\#\left(\widetilde{\omega}_{N} \cap B\right)}{N} & =\frac{\bar{g}_{\infty, \lambda}(B)^{\lambda}}{\bar{g}_{\infty, \lambda}(B)^{\lambda}+\bar{g}_{\infty, \lambda}(A \backslash B)^{\lambda}} \\
& =\frac{h_{\lambda, K}(\bar{B})}{h_{\lambda, K}(\bar{B})+h_{\lambda, K}(\overline{A \backslash B})}=h_{\lambda, A}(B),
\end{aligned}
$$

which completes the proof of relations (19) and (20). Corollary 1 is proved.

\section{Proof of Theorem 3}

To prove Theorem 3 in the case $s \in(\alpha, \infty)$, we will need Lemma 2 and in the case $s=\infty$, we will use its analogue given below.

Lemma 6. Let $m \in \mathbb{N}, 0<\alpha \leq m$, and $K$ be a compact set in $\mathbb{R}^{m}$ with $P^{\alpha}(K)<\infty$. Then for every compact subset $F \subset K$ we have

$$
\bar{g}_{\infty, \alpha}(F)=\inf _{\substack{U \supset F \\ U \text {-open }}} \bar{g}_{\infty, \alpha}(K \cap U) .
$$

Proof of Lemma 6: Let

$$
C_{0}:=\inf _{\substack{U \supset F \\ U \text {-open }}} \bar{g}_{\infty, \alpha}(K \cap U) .
$$


Clearly, $\bar{g}_{\infty, \alpha}(F) \leq C_{0}$. Assume to the contrary that $\bar{g}_{\infty, \alpha}(F)<C_{0}$. This will imply that at least $C_{0}>0$. Choose any $\epsilon \in\left(0,\left(C_{0} / 8\right)^{2 \alpha}\right)$ and let $U_{\epsilon}$ be the set from Lemma 1 . Denote by $\mathcal{N}$ an infinite subset of $\mathbb{N}$ such that

$$
\lim _{\mathcal{N} \ni N \rightarrow \infty} \delta_{N}\left(\overline{K \cap U_{\epsilon}}\right) N^{1 / \alpha}=\bar{g}_{\infty, \alpha}\left(\overline{K \cap U_{\epsilon}}\right) \geq C_{0}>0,
$$

and let $\omega_{N}^{*}=\left\{x_{1, N}, \ldots, x_{N, N}\right\}, N \in \mathcal{N}$, be a sequence of best-packing configurations on $\overline{K \cap U_{\epsilon}}$. Note that for any $N \in \mathcal{N}$ sufficiently large and any $x \neq y \in \omega_{N}^{*}$ we have $|x-y| \geq \frac{C_{0}}{2} N^{-1 / \alpha}$. Denote $\rho:=\epsilon^{\frac{1}{2 \alpha}}$ and let

$$
\omega_{N}^{\prime}:=\left\{x \in \omega_{N}^{*}: \operatorname{dist}(x, F)<2 \rho N^{-1 / \alpha}\right\} .
$$

Applying Proposition 1 with $G=F, H=\overline{K \cap U_{\epsilon}}, X_{M}=\omega_{N}^{*}, Y_{M}=\omega_{N}^{\prime}$ and $\delta=2 \rho N^{-1 / \alpha}$, we obtain that for $N$ sufficiently large

$$
\#\left(\omega_{N}^{*} \backslash \omega_{N}^{\prime}\right) \leq 3 \epsilon \rho^{-\alpha} N=3 \epsilon^{1 / 2} N
$$

and hence,

$$
\# \omega_{N}^{\prime} \geq\left(1-3 \epsilon^{1 / 2}\right) N=: N_{\epsilon} .
$$

For every $x \in \omega_{N}^{\prime}$ let $y_{x}$ be an element in $F$ such that $\operatorname{dist}\left(x, y_{x}\right)<$ $2 \rho N^{-1 / \alpha}$. Denote $\omega_{F}:=\left\{y_{x}: x \in \omega_{N}^{\prime}\right\}$. Note that for every $x \neq z \in \omega_{N}^{\prime}$, and $N \in \mathcal{N}$ sufficiently large, we have $|x-z| \geq \frac{C_{0}}{2} N^{-1 / \alpha}$ and

$$
\begin{aligned}
\left|y_{x}-y_{z}\right| & \geq|x-z|-\left|x-y_{x}\right|-\left|z-y_{z}\right| \\
& \geq|x-z|-4 \rho N^{-1 / \alpha} \geq\left(1-8 \rho / C_{0}\right)|x-z|>0 .
\end{aligned}
$$

Here we have $8 \rho<C_{0}$ by the choice of $\epsilon$. Then we get that $\# \omega_{F}=\# \omega_{N}^{\prime}$ and

$$
\begin{aligned}
C_{0} & \leq \bar{g}_{\infty, \alpha}\left(\overline{K \cap U_{\epsilon}}\right)=\lim _{\mathcal{N} \ni N \rightarrow \infty} \delta_{N}\left(\overline{K \cap U_{\epsilon}}\right) \cdot N^{1 / \alpha} \\
& =\lim _{\mathcal{N} \ni N \rightarrow \infty} \delta\left(\omega_{N}^{*}\right) \cdot N^{1 / \alpha} \leq \limsup _{\mathcal{N} \ni N \rightarrow \infty} \delta\left(\omega_{N}^{\prime}\right) \cdot N^{1 / \alpha} \\
& \leq\left(1-8 \rho / C_{0}\right)^{-1} \limsup _{\mathcal{N} \ni N \rightarrow \infty} \delta\left(\omega_{F}\right) \cdot N^{1 / \alpha} \\
& \leq\left(1-8 \rho / C_{0}\right)^{-1}\left(1-3 \epsilon^{1 / 2}\right)^{-1 / \alpha} \limsup _{\mathcal{N} \ni N \rightarrow \infty} \delta_{\left[N_{\epsilon}\right]}(F) \cdot\left[N_{\epsilon}\right]^{1 / \alpha} \\
& \leq\left(1-8 \rho / C_{0}\right)^{-1}\left(1-3 \epsilon^{1 / 2}\right)^{-1 / \alpha} \bar{g}_{\infty, \alpha}(F) .
\end{aligned}
$$

Letting $\epsilon \rightarrow 0$ we get that $C_{0} \leq \bar{g}_{\infty, \alpha}(F)$ which contradicts to our assumption. Hence, $C_{0}=\bar{g}_{\infty, \alpha}(F)$. Lemma 6 is proved. 
To complete the proof of Theorem 3 , let $s \in[a, \infty]$ and define

$$
g(A)= \begin{cases}\underline{g}_{s, \alpha}(A)^{-\alpha / s}, & \alpha<s<\infty, \\ \bar{g}_{\infty, \alpha}(A)^{\alpha}, & s=\infty .\end{cases}
$$

The statement below is contained in Lemma 3 for $s<\infty$ and is proved in [19] or [6] for $s=\infty$. In the case $s=\infty$ one can obtain the assertion of Lemma 7 by letting $s \rightarrow \infty$ in Lemma 3 and applying (6).

Lemma 7. Let $m \in \mathbb{N}, 0<\alpha \leq m, s \in(\alpha, \infty]$ and $B$ and $D$ be any sets in $\mathbb{R}^{m}$. Then

$$
g(B \cup D) \leq g(B)+g(D) .
$$

Choose again any $\epsilon>0$. Let $\left\{A_{i}\right\}_{i \in \mathbb{N}}$ be a collection of subsets of $A$ such that $A=\cup_{i \in \mathbb{N}} A_{i}$ and

$$
\sum_{i \in \mathbb{N}} g\left(A_{i}\right)<\nu_{s, \alpha}(A)+\epsilon .
$$

Let now $\mathcal{N} \subset \mathbb{N}$ be the set of indices $i$ such that $A_{i} \neq \emptyset$. For every $i \in \mathcal{N}$, let $U_{i, \epsilon}$ be an open set in $\mathbb{R}^{m}$ such that $\bar{A}_{i} \subset U_{i, \epsilon}$ and

$$
g\left(A \cap U_{i, \epsilon}\right)<g\left(\bar{A}_{i}\right)+\epsilon / 2^{i}=g\left(A_{i}\right)+\epsilon / 2^{i} .
$$

The existence of $U_{i, \epsilon}$ is guaranteed by Lemma 2 for $s<\infty$ and by Lemma 6 for $s=\infty$. Since the collection $\left\{U_{i, \epsilon}\right\}_{i \in \mathcal{N}}$ covers $A$, it has a finite subcollection $\left\{U_{i_{k}, \epsilon}\right\}_{k=1}^{p}$ that still covers $A$. Then using Lemma 7 and (35) we get

$$
\begin{aligned}
g(A) & =g\left(\bigcup_{k=1}^{p}\left(A \cap U_{i_{k}, \epsilon}\right)\right) \leq \sum_{k=1}^{p} g\left(A \cap U_{i_{k}, \epsilon}\right) \\
& \leq \sum_{k=1}^{p}\left(g\left(A_{i_{k}}\right)+\epsilon / 2^{i_{k}}\right) \leq \sum_{i=1}^{\infty} g\left(A_{i}\right)+\epsilon<\nu_{s, \alpha}(A)+2 \epsilon .
\end{aligned}
$$

In view of arbitrariness of $\epsilon$, we get $g(A) \leq \nu_{s, \alpha}(A), s \in(\alpha, \infty]$. The opposite inequality is obvious. Theorem 3 is proved.

Acknowledgements. The author would like to express his gratitude to Professor Pertti Mattila for an opportunity to visit at the University of Helsinki and many interesting and fruitful discussions which helped in working over this paper. 


\section{References}

[1] V. Afraimovich and L. Glebsky, Measures of $\epsilon$-complexity, Taiwanese J. Math. 9(3) (2005), 397-409.

[2] V. Afraimovich and L. Glebsky, Measures related to $(\epsilon, n)$ complexity functions, Discrete Contin. Dyn. Syst. 22(1-2) (2008), 23-34. DOI: $10.3934 /$ dcds.2008.22.23.

[3] N. N. Andreev, Location of points on a sphere with minimal energy, (Russian), Tr. Mat. Inst. Steklova 219, Teor. Priblizh. Garmon. Anal. (1997), 27-31; translation in: Proc. Steklov Inst. Math. 219(4) (1997), 20-24.

[4] K. BÖRÖCZKY, JR., "Finite packing and covering", Cambridge Tracts in Mathematics 154, Cambridge University Press, Cambridge, 2004. DOI : 10.1017/CB09780511546587.

[5] S. V. Borodachov, D. P. Hardin, and E. B. Saff, Asymptotics for discrete weighted minimal Riesz energy problems on rectifiable sets, Trans. Amer. Math. Soc. 360(3) (2008), 1559-1580 (electronic). DOI : 10.1090/S0002-9947-07-04416-9.

[6] S. V. Borodachov, D. P. Hardin, and E. B. Saff, Asymptotics of weighted best-packing on rectifiable sets, Sb. Math. 199(11) (2008), 1579-1595.

DOI: $10.1070 /$ SM2008v199n11ABEH003973.

[7] S. V. Borodachov, D. P. Hardin, and E. B. Saff, Asymptotics of best-packing on rectifiable sets, Proc. Amer. Math. Soc. 135(8) (2007), 2369-2380 (electronic). DOI : 10.1090/S0002-993907-08975-7.

[8] H. Cohn And N. Elkies, New upper bounds on sphere packings, I, Ann. of Math. (2) 157(2) (2003), 689-714. DOI: 10.4007/annals. 2003.157.689.

[9] H. Cohn And A. Kumar, Universally optimal distribution of points on spheres, J. Amer. Math. Soc. 20(1) (2007), 99-148. DOI: 10.1090/S0894-0347-06-00546-7.

[10] J. H. Conway and N. J. A. Sloane, "Sphere packings, lattices and groups", Third edition, Grundlehren der Mathematischen Wissenschaften 290, Springer-Verlag, New York, 1999.

[11] K. J. FALCONER, "The geometry of fractal sets", Cambridge Tracts in Mathematics 85, Cambridge University Press, Cambridge, 1986.

[12] H. Federer, "Geometric measure theory", Die Grundlehren der mathematischen Wissenschaften 153, Springer-Verlag New York Inc., New York, 1969. 
[13] L. Fejes Tóth, "Regular figures", A Pergamon Press Book, The Macmillan Co., New York, 1964.

[14] D.-J. Feng, S. HuA, And Z.-Y. Wen, Some relations between packing premeasure and packing measure, Bull. London Math. Soc. 31(6) (1999), 665-670. DOI : 10.1112/S0024609399006256.

[15] S. P. Ferguson, Sphere packings. V. Pentahedral prisms, Discrete Comput. Geom. 36(1) (2006), 167-204. DOI: 10.1007/s00454-0051214-y.

[16] P. Grabner, D. P. Hardin, and E. B. Saff, Discrete and continuous singular minimal energy on self-similar fractals, International Conference "Optimal Configurations on the Sphere and other Manifolds", Vanderbilt University, May 17-20, 2010.

[17] S. GraF AND H. LuschGy, "Foundations of quantization for probability distributions", Lecture Notes in Mathematics 1730, SpringerVerlag, Berlin, 2000. DOI: 10.1007/BFb0103945.

[18] S. Graf And H. Luschgy, The point density measure in the quantization of self-similar probabilities, Math. Proc. Cambridge Philos. Soc. 138(3) (2005), 513-531. DOI: 10.1017/S030500410500842X.

[19] H. HAAse, A contribution to measure and dimension of metric spaces, Math. Nachr. 124 (1985), 45-55. DOI: 10.1002/mana. 19851240104.

[20] H. HAASE, Metric outer measures of type I in the general setting, Math. Nachr. 135 (1988), 35-40. DOI: 10.1002/mana.19881350103.

[21] T. C. Hales, A proof of the Kepler conjecture, Ann. of Math. (2) 162(3) (2005), 1065-1185. DOI: 10.4007/annals.2005.162.1065.

[22] D. P. HARdin And E. B. SAFF, Discretizing manifolds via minimum energy points, Notices Amer. Math. Soc. 51(10) (2004), 1186-1194.

[23] D. P. Hardin And E. B. SAFF, Minimal Riesz energy point configurations for rectifiable $d$-dimensional manifolds, Adv. Math. 193(1) (2005), 174-204. DOI: 10.1016/j.aim.2004.05.006.

[24] E. Hewitt and K. Stromberg, "Real and abstract analysis. A modern treatment of the theory of functions of a real variable", Springer-Verlag, New York, 1965.

[25] J. E. Hutchinson, Fractals and self-similarity, Indiana Univ. Math. J. 30(5) (1981), 713-747. DOI : 10.1512/iumj.1981.30.30055.

[26] A. N. Kolmogorov, On certain asymptotic characteristics of completely bounded metric spaces, (Russian), Dokl. Akad. Nauk SSSR (N.S.) 108 (1956), 385-388.

[27] A. N. Kolmogorov And V. M. Tikhomirov, $\varepsilon$-entropy and E-capacity of sets in functional space, (Russian), Uspehi Mat. 
Nauk (N.S.) 14(2) (1959), 3-86: translation in: Amer. Math. Soc. Transl. (2) 17 (1961), 277-364.

[28] A. V. Kolushov And V. A. Yudin, Extremal dispositions of points on the sphere, Anal. Math. 23(1) (1997), 25-34. DOI: 10.1007/BF02789828.

[29] S. P. LALleY, The packing and covering functions of some selfsimilar fractals, Indiana Univ. Math. J. 37(3) (1988), 699-710. DOI: 10.1512/iumj.1988.37.37034.

[30] N. S. LANDKOF, "Foundations of modern potential theory", Translated from the Russian by A. P. Doohovskoy, Die Grundlehren der mathematischen Wissenschaften 180, Springer-Verlag, New YorkHeidelberg, 1972.

[31] A. Martínez-Finkelshtein, V. Maymeskul, E. A. Rakhmanov, AND E. B. SAFF, Asymptotics for minimal discrete Riesz energy on curves in $\mathbb{R}^{d}$, Canad. J. Math. 56(3) (2004), 529-552. DOI : $10.4153 / \mathrm{CJM}-2004-024-1$.

[32] P. Mattila, "Geometry of sets and measures in Euclidean spaces", Fractals and rectifiability, Cambridge Studies in Advanced Mathematics 44, Cambridge University Press, Cambridge, 1995.

[33] M. Morán, Computability of the Hausdorff and packing measures on self-similar sets and the self-similar tiling principle, Nonlinearity 18(2) (2005), 559-570. DOI: 10.1088/0951-7715/18/2/006.

[34] P. A. P. Moran, Additive functions of intervals and Hausdorff measure, Proc. Cambridge Philos. Soc. 42 (1946), 15-23.

[35] L. Pontruagin and L. Schnirelmann, Sur une propriété métrique de la dimension, Ann. of Math. (2) 33(1) (1932), 156-162. DOI: 10.2307/1968109.

[36] K. Pötzelberger, The general quantization problem for distributions with regular support, Math. Methods Statist. 9(2) (2000), 176-198.

[37] C. A. Rogers, "Packing and covering", Cambridge Tracts in Mathematics and Mathematical Physics 54, Cambridge University Press, New York, 1964.

[38] A. Schief, Separation properties for self-similar sets, Proc. Amer. Math. Soc. 122(1) (1994), 111-115. DOI: 10.2307/2160849.

[39] D. Sullivan, Entropy, Hausdorff measures old and new, and limit sets of geometrically finite Kleinian groups, Acta Math. 153(3-4) (1984), 259-277. DOI: 10.1007/BF02392379.

[40] S. J. TAylor and C. Tricot, Packing measure, and its evaluation for a Brownian path, Trans. Amer. Math. Soc. 288(2) (1985), 679-699. DOI: 10.2307/1999958. 
[41] S. J. TAYlor and C. TRicot, The packing measure of rectifiable subsets of the plane, Math. Proc. Cambridge Philos. Soc. 99(2) (1986), 285-296. DOI: 10.1017/S0305004100064203.

[42] C. Tricot, JR., Two definitions of fractional dimension, Math. Proc. Cambridge Philos. Soc. 91(1) (1982), 57-74. DOI: 10.1017/ S0305004100059119.

[43] V. A. Yudin, Minimum potential energy of a point system of charges, (Russian), Diskret. Mat. 4(2) (1992), 115-121; translation in: Discrete Math. Appl. 3(1) (1993), 75-81. DOI: 10.1515/dma. 1993.3.1.75.

[44] O. Zindulka, Packing measures and Cartesian products, Real Anal. Exchange, 31st Summer Symposium Conference (2007), 195-200.

Department of Mathematics

Towson University

Towson, Baltimore MD 21252

USA

E-mail address: SBorodachov@towson.edu

Primera versió rebuda el 31 de gener de 2011, darrera versió rebuda el 5 de juliol de 2011 . 